\title{
Les ingénieurs parlementaires et la question des réseaux secondaires au début de la Troisième République (années 1870-1880)
}

\section{Bruno Marnot}

\section{CpenEdition}

\section{Journals}

Édition électronique

URL : https://journals.openedition.org/rhcf/2033

DOI : 10.4000/rhcf.2033

Éditeur

Rails \& histoire

Édition imprimée

Date de publication : 2 mai 2002

Pagination : 89-10

ISBN : 00996-9403

ISSN : 0996-9403

Référence électronique

Bruno Marnot, «Les ingénieurs parlementaires et la question des réseaux secondaires au début de la Troisième République (années 1870-1880) », Revue d'histoire des chemins de fer [En ligne], 24-25 | 2002, mis en ligne le 03 février 2015, consulté le 22 avril 2022. URL : http://journals.openedition.org/rhcf/ 2033 ; DOI : https://doi.org/10.4000/rhcf.2033 


\section{Les ingénieurs parlementaires et la question des réseaux secondaires au début de la Troisième République (années 1870-1880)}

La question des réseaux secondaires est l'une des controverses majeures qui agite le milieu des ingénieurs parlementaires, dont le rôle dans l'élaboration de la politique ferroviaire fut essentiel dès les premières années de la Troisième République. Avant même de définir les enjeux du débat, deux points doivent être abordés pour éclairer notre propos.

Le premier concerne le profil socioprofessionnel des ingénieurs élus : sur une étude plus vaste de l'évolution électorale des ingénieurs parlementaires, qui couvrait les quelque soixante-dix années de la Troisième République, une première tranche chronologique se dégageait nettement avec la période 1871-1889. Au cours de ces années, 94 ingénieurs ont détenu un mandat national mais ce qui importe surtout, en l'occurrence, c'est la forte proportion, jamais revue par la suite, d'ingénieurs d'État, qui constituent $36 \%$ du corpus. Plus intéressant encore, ce sous-ensemble révèle la prépondérance du corps des Ponts et Chaussées qui représente les deux tiers des ingénieurs d'État présents au Parlement. Ces précisions liminaires sont essentielles pour apprécier la teneur des réflexions exposées par cette catégorie d'élus : ce sont essentiellement les ingénieurs d'État, et plus particulièrement ceux des Ponts, qui animent les débats relatifs aux réseaux secondaires ; cela signifie que les ingénieurs civils, représentés de manière quasi exclusive par des centraliens au cours de cette période, traditionnellement favorables aux lignes d'intérêt local et adversaires des grandes compagnies, restent à peu près muets. Le débat est par conséquent d'emblée orienté.

Le deuxième point concerne la période même de notre étude, c'est-à-dire de 1871 au milieu des années 1880, marquée par quelques dates et événements-clés : le lancement des « lignes Caillaux » de 1875 ; le rachat de lignes secondaires en 1878 et la fin du « système Philippart »; le plan Freycinet de 1879 ; enfin, la loi de 1880 qui remédie aux lacunes de la loi de 1865. Cette décennie est par conséquent marquée par une volonté manifeste d'étoffer le réseau français, ce que certains appellent, 
au cours de ces années, son «achèvement", qui passe désormais par le développement de la vicinalité ferroviaire. Ces années apparaissent comme cruciales pour l'avenir des réseaux secondaires, et leur sont en fait plutôt favorables dans un contexte marqué par une critique ouverte et virulente à l'encontre des grandes compagnies.

Le problème est posé de manière claire par les ingénieurs. Quelle est l'utilité de tels chemins de fer? C'est une question centrale, constamment reposée dès qu'on aborde la question des transports au $\mathrm{XIX}^{\mathrm{e}}$ siècle. C'est dire si les réflexions émises par les ingénieurs parlementaires ne sont pas nouvelles, puisqu'elles ressuscitent des questionnements soulevés au moment de la loi de 1865, mais le contexte politique et économique des années 1870 et 1880 leur donnent une nouvelle vigueur.

La question de l'utilité recouvre trois aspects. Un enjeu politicoéconomique d'abord, enjeu majeur et central auquel sont subordonnées toutes les autres questions et controverses ; un débat technique ensuite, portant sur les caractéristiques de la voie d'intérêt local ; la question financière, enfin, puisque l'une des principales qualités de ces réseaux réside dans l'économie de leur établissement et de leur exploitation.

\section{L'enjeu politico-économique}

En 1875, paraissent aux éditions Delamotte et fils deux opuscules signés de la main de Jean-Baptiste Krantz. Le premier s'intitule: Observations au sujet des chemins de fer d'intérêt général et local; le second: Observations sur les chemins de fer économiques à voie normale et à voie réduite. Qui est Krantz ? Il est sans doute l'un des personnages les plus éminents de la phalange technicienne présente au Parlement. Vosgien d'origine, membre du corps des Ponts et Chaussées, il a effectué l'essentiel de sa carrière sous la monarchie de Juillet et le Second Empire, carrière brillante dont les faits saillants furent la supervision de la construction du port d'Alger entre 1842 et 1853 ; le passage dans le secteur privé pendant une décennie, de 1853 à 1863, au service des compagnies ferroviaires du Grand Central puis du Paris-Orléans ; l'obtention, en guise de couronnement, du commissariat général à l'Exposition universelle de 1878. C'est là une preuve de reconnaissance de la jeune République à l'égard de l'un de ses grands commis, homme du centre gauche qui représente la Seine à l'Assemblée nationale en 1871, avant de devenir l'un des 116 sénateurs inamovibles élus en $1875^{1}$.

A l'Assemblée, et pour la postérité, Krantz est surtout connu comme le grand défenseur des voies navigables et de la cause batelière, comme l'ont bien montré les travaux de M. Merger ${ }^{2}$. On oublie qu'il 
fut aussi l'un des acteurs politiques les plus engagés dans la réflexion sur l'avenir des chemins de fer en France et, plus particulièrement, sur la place et la fonction à attribuer aux réseaux secondaires. Que les deux ouvrages cités plus haut soient publiés en 1875 n'est pas pur hasard. Cette année marque un tournant dans l'histoire ferroviaire de la Troisième République, puisque le ministre des Travaux publics, Eugène Caillaux, un autre membre du corps des Ponts et Chaussées, dépose deux projets de loi portant sur le classement de plus de 1200 kilomètres de lignes nouvelles, soit respectivement 396 kilomètres situés dans l'Ouest et 896 autres kilomètres de lignes dispersées dans le centre de la France et le Sud-Ouest, dans le but de multiplier les liaisons transversales. Après les années 1871-1874 qui ont vu la victoire progressive des partisans du système ferroviaire français élaboré sous les deux régimes précédents et fondé sur les relations étroites entre l'État et les grandes compagnies, l'année 1875 correspond bien à la relance de la vicinalité ferroviaire, car la "soif universelle des chemins de fer", pour reprendre l'expression désormais canonique de L. Girard, est loin d'être assouvie, comme le confirme quelques années plus tard le plan Freycinet.

A lire attentivement les projets de loi votés en décembre 1875, on ne peut s'empêcher de relever que la construction et l'exploitation des nouvelles lignes ne doivent pas être confiées, dans l'esprit d'un ministre entièrement dévolu à la cause des grandes compagnies, à des intérêts locaux dont il redoute la faible assise financière et qui sont souvent les victimes de bailleurs de fonds malhonnêtes. C'est en effet la conséquence la plus funeste des carences de la loi de 1865. La réflexion de Krantz s'inscrit donc dans ce contexte : un évident souci de renforcer le maillage ferroviaire sur le territoire national, mais par quels voies et moyens?

Dès l'avant-propos des Observations au sujet des chemins de fer d'intérêt général et local, Krantz évoque l'atmosphère de « conflit » dont l'origine est à rechercher dans les lois de 1865 et 1871, lesquelles « peuvent, suivant les influences dominantes, donner naissance soit à l'arbitraire administratif, soit à l'anarchie industrielle». Il dénonce notamment la «naïveté des conseils généraux » qui « ne se sont pas toujours exactement rendu compte des engagements que leur imposent les concessions $[\ldots] »^{3}$.

Est-ce à dire que cet homme des Ponts et Chaussées, cet ancien ingénieur en chef de grandes compagnies privées, ce défenseur de la voie d'eau s'oppose à l'extension du réseau ferroviaire qui passe désormais par la construction de voies secondaires? En fait, Krantz est favorable à la densification du réseau national, mais en la plaçant sous le sceau de «l'utilité générale des chemins de fer », concept central, 
riche et objet d'ardentes polémiques théoriques depuis l'apparition de ce nouveau mode de transport. Pour Krantz, l'utilité ne se réduit pas à sa seule acception économique, même si «l'accroissement d'activité et de richesses $»^{4}$ qui résultent du développement des chemins de fer ne fait pas l'ombre d'un doute. Deux autres motifs justifient l'établissement de voies secondaires. Le premier relève de la rhétorique saint-simonienne, puisque cet ingénieur - qui défend au cours de la même année, à l'Assemblée, un projet de loi gouvernemental de tunnel sous-marin entre la France et l'Angleterre - rappelle «l'influence civilisatrice » des voies de communication ${ }^{5}$. Le deuxième est d'une tonalité plus républicaine puisqu'il voit dans l'achèvement du réseau une « question de justice $\|^{6}$, c'est-à-dire l'accomplissement d'une forme d'égalité et de solidarité civiques nouvelles par l'accès universel - en fait celui des populations rurales - à un moyen de transport moderne dont l'édification exige un effort financier collectif.

«L'achèvement » du réseau : l'expression fait florès au cours des années suivantes, au point de devenir le leitmotiv des premiers gouvernements authentiquement républicains, et elle signifie concrètement l'irrigation de toutes les contrées du territoire par des réseaux secondaires. Quant à définir précisément les limites kilométriques de cet achèvement, les spéculations vont bon train : Krantz, qui ne manque pas de souligner les vides du Centre et de l'Ouest, estime que 20000 nouveaux kilomètres sont encore nécessaires, soit à peu près le doublement du réseau français ; en 1881, un autre ingénieur des Ponts parlementaire, Jules Lesguillier, propose pas moins de 30000 kilomètres supplémentaires. C'est en tout cas cette conception de l'utilité, dont se départira d'ailleurs Krantz quelques années plus tard, qui inspire la construction du «troisième réseau » en 1879, conception partagée par d'autres ingénieurs d'État, également parlementaires et appelés à jouer un rôle de premier plan au ministère des Travaux publics, tels que Freycinet ou Henri Varroy.

Si, en 1875, Krantz est convaincu de l'utilité des chemins de fer secondaires, il ne pose pas moins la question cruciale de leur articulation, au sens technique et conceptuel, avec les lignes d'intérêt général. Le dilemme est simple dans son énoncé : les voies d'intérêt local sont-elles appelées à devenir des concurrentes des lignes d'intérêt général, comme le souhaitent depuis longtemps les partisans de la décentralisation et des libertés locales, ou, au contraire, doit-on les contenir dans leur « rôle unique d'affluent $»^{7}$, ainsi que le supposerait une vision globale et hiérarchisée du réseau français? Cette alternative recouvre en fait deux interrogations d'ordre économique : les lignes d'intérêt local sont-elles capables d'engendrer un trafic suffisant pour survivre ? Et, si cette première condition est remplie, cela ne risque-t-il pas d'affecter l'activité 
des grandes compagnies en détournant une partie du produit des anciennes lignes? A cette double interrogation, Krantz répond, il faut bien le dire, de manière évasive, convaincu que la multiplication des lignes engendrera de facto de l'activité et par conséquent du trafic, tant et si bien que la cohabitation des grands et petits réseaux est possible. "C'est une quantité variable qui peut croître dans une large mesure, affirme-t-il, à condition que le travail, l'industrie, les capitaux se développent, et surtout que des voies de communication faciles permettent d'écouler dans tous les sens les produits créés. On voit donc que les craintes des grandes compagnies ne sont pas toujours fondées et que leur opposition aux nouvelles créations de chemins de fer est quelquefois excessive ${ }^{8}$ »

Pour Krantz, la nécessité de développer un réseau d'intérêt local ne fait pas de doute, mais il faut modifier la procédure. En premier lieu, il revient au pouvoir central d'établir un classement des lignes à construire en fonction, d'une part, des revendications départementales, d'autre part, de leur réel intérêt économique, enfin, des ressources financières dont on dispose. Il faut ensuite en finir avec cette pernicieuse législation de 1865 qui a surtout attisé l'appétit des spéculateurs. Il convient par conséquent de redonner un réel pouvoir de décision aux conseils généraux ainsi qu'aux intéressés, de même qu'une autonomie financière en les dotant d'une « équitable subvention »". Il faut d'ailleurs noter que Krantz ne dit rien de la nature et des conditions de versement de cette subvention. Il prône, en tout cas, la constitution de syndicats de départements ${ }^{10}$ qui représenteraient, selon lui, le seuil de viabilité financière pour ce type de réseau. La procédure décisionnelle serait la suivante : l'État autoriserait les syndicats de départements à concéder les lignes dont il aurait approuvé par avance les cahiers des charges. Il se réserverait la faculté de les racheter à toute époque, avant l'expiration des quinze premières années d'exploitation, en remboursant les frais de premier établissement. «De cette façon, conclut Krantz, les lignes réservées se feraient aussi vite que les autres et les intérêts que l'État veut sauvegarder n'auraient aucun risque à couriri ${ }^{11}$. » L'ingénieur maintient bien le principe d'une déclaration d'utilité publique mais il ne dit pas qui, du pouvoir exécutif ou du pouvoir législatif, détiendrait l'autorité pour l'accorder. La loi du 11 juin 1880 sur les chemins de fer d'intérêt local et les tramways apporte deux innovations essentielles : la déclaration d'utilité publique et l'autorisation d'exécution sont transférées au Parlement ; les subventions en capital versées par le Trésor sont remplacées par des subventions annuelles distribuées après la mise en exploitation des lignes. Tout danger de spéculation est ainsi théoriquement écarté1 ${ }^{12}$. 
Krantz s'efforce donc de trouver un point d'équilibre entre l'intérêt général et les intérêts des départements, comme il souhaite établir une complémentarité entre les grandes lignes et les réseaux secondaires. La distinction devient néanmoins difficile à opérer dès qu'on aborde la notion de « réseau » de transport. La loi de 1865 souffre en effet d'une lacune originelle beaucoup plus gênante que les effets délétères dénoncés plus haut, car il y manque, selon l'ingénieur, une définition exacte du chemin de fer d'intérêt local. Et Krantz de constater que « ni la loi en son texte formel, ni les commentateurs officiels de la loi dans leurs rapports et circulaires, ni la langue en ses acceptions reçues, ni l'étude attentive des réseaux actuels, ne permettent de reconnaitre d'une manière certaine, indiscutable, si un chemin est d'intérêt général ou local $»^{13}$. Cette remarque pose le problème de la signification concrète de la voie d'intérêt local. Aussi Krantz en vient-il à cette ultime interrogation : dans la mesure où l'établissement de réseaux secondaires ne saurait être envisagé sans leur raccordement au réseau d'intérêt général, « le mot chemin d'intérêt local ne saurait équivaloir à une définition et n'est même pas complètement juste ; car enfin, s'il est de l'intérêt d'une localité de faire arriver aisément ses produits à une grande voie de fer qui les transporte au loin, il est aussi de l'intérêt des destinataires éloignés de recevoir ces produits dans de meilleures conditions de prix et de célérité : intérêt local d'un côté, général de l'autre $»^{14}$. Cette dernière phrase est essentielle. Si Krantz conçoit la légitimité d'une distinction théorique, en tout cas pertinente pour l'administration et le législateur, entre les deux types de réseaux, dans la réalité une séparation aussi tranchée n'aurait aucun sens : intérêt local et intérêt général s'interpénètrent constamment, et l'on peut même se risquer à dire, si l'on force un peu la pensée de Krantz, que pour une localité désireuse de développer son commerce avec l'extérieur, il n'y a d'intérêt que général puisque ses produits sont nécessairement appelés à emprunter les lignes du même nom.

\section{Le débat technique}

Il est alors évident que l'articulation entre réseau d'intérêt local et réseau d'intérêt général constitue le cœur du débat technique. C'est tout le problème de la continuité du réseau et, par conséquent, de sa fluidité, qui est posé. Si le principe de la voie d'intérêt local ne convainc pas tous les ingénieurs parlementaires, ses partisans ne parviennent pas pour autant à établir un front commun, à cause de la diversité des alternatives proposées entre chemins de fer sur route ou en site propre, voie à écartement réduit ou "normal », ou encore voie unique ou double voie. 
Le principe du chemin de fer établi sur route rencontre en fait peu de partisans parmi les ingénieurs du Parlement. Qu'il soit ou non à écartement réduit, il éveille plus de circonspection que d'enthousiasme. Dans son deuxième ouvrage publié en 1875, Krantz affiche son scepticisme à l'égard d'une voie installée sur l'accotement, source de difficultés pour le stationnement des voitures qui se chargent ou se délestent de leurs produits. Au risque d'encombrement s'ajoute l'obstacle de pentes et de courbes inadaptées à la voie ferrée. Ce système hybride doit donc être rejeté au nom d'une séparation rationnelle des circulations ${ }^{15}$. Un projet de loi relatif «aux chemins de fer à traction de locomotives pouvant être établis sur les routes » est néanmoins déposé le 17 mars 1875 par le ministre Caillaux dans le but avoué de faire de «notables économies ». Le ministre des Travaux publics, au cours d'une intervention en séance publique, considérant à son terme l'achèvement des projets de grandes lignes du réseau français, plaide en faveur d'un effort vis-àvis du réseau vicinal qu'il est urgent d'établir «à voie réduite toujours, et, lorsqu'on le pourra sur les accotements des routes, afin de ne pas dépenser les sommes considérables qu'on gaspille si souvent inutilement dans les conditions actuelles $»^{16}$. La commission ad hoc désigne Varroy comme rapporteur, lequel fait preuve de réserve, comme l'ensemble de ses collègues. En outre, le rapporteur signale l'absence de consensus chez les techniciens à propos de l'adoption de la voie étroite sur de tels chemins. Il en veut pour preuve «les tramways à traction de chevaux, qui commencent à se propager dans nos villes, [et qui] loin de rechercher l'économie que l'on attribue à l'étroitesse de la voie, adoptent généralement, avec des rails légers, l'écartement normal de 1,44 mètre, pour se ménager la possibilité de faire circuler dans nos rues les wagons de marchandises des compagnies de chemins de fer $\rangle^{17}$. Varroy redoute plus que tout la rupture de charge qui multiplie les manutentions et, donc, les pertes de temps. En fait, le seul des ingénieurs parlementaires à défendre cette version de la voie d'intérêt local est le député radical de l'Aude et ingénieur des Mines, Emile Wickersheimer, car il la considère comme représentative des « chemins de fer véritablement ruraux $»^{18}$.

A l'instar de Varroy, Krantz considère que le chemin de fer d'intérêt local doit être construit à l'écartement normal. Sa doctrine lui est d'ailleurs inspirée par son collègue des Ponts qui a rédigé une Note sur les chemins de fer départementaux ou d'intérêt local qu'il qualifie dans un élan d'enthousiasme de "véritable modèle de fine et puissante analyse $»^{19}$. En fait, le propos de Krantz ne consiste pas tant à imposer le principe de la voie unique à écartement normal qu’à démontrer les inconvénients de la voie étroite 
considérée par d'aucuns comme la véritable voie d'intérêt local ${ }^{20}$. Ainsi, les qualités de flexibilité attribuées à la voie étroite sont discutables. Concernant les rampes, la voie d'un mètre ne s'avère pas supérieure à la voie de 1,44 mètre ; concernant les courbes, la première est supérieure à la seconde seulement dans les rayons de moins de 150 mètres. Dans le même ordre d'idées, Krantz relativise la théorie du rail léger sur voie étroite qui permettrait de réaliser de substantielles économies. Il considère le trafic comme une variable beaucoup plus déterminante: «si une ligne de faible largeur possède un trafic développé, on arrivera infailliblement à y employer des rails puissants ${ }^{21}$. »

Le grief essentiel exprimé par Krantz n'est pourtant pas là. Le risque majeur de la voie métrique réside dans la multiplication des transbordements qu'elle entraîne inéluctablement et qui relèvent selon lui de "l'anachronisme économique $»^{22}$. C'est un jugement sans appel d'une doctrine qui lui parait relever de la pure aberration technique et économique. Il parle ainsi au nom des commerçants et des industriels qui " ont une aversion très prononcée contre les transbordements »" onéreux et causes de ralentissement. Au même titre qu'il récuse la diversité des types d'écluses en matière de navigation fluviale, Krantz définit une conception des transports assise sur la standardisation des infrastructures, seule garantie de continuité et de fluidité des circulations, donc en définitive seule condition de leur efficacité. Dans la continuité des principes énoncés dans son autre ouvrage, Krantz défend la voie normale au nom de l'indispensable homogénéité du réseau ${ }^{24}$. Enfin, l'ingénieur des Ponts apporte à son plaidoyer un ultime et pesant argument, d'ordre pécuniaire, puisqu'il prétend que la construction et l'exploitation de lignes d'intérêt local à voie normale peuvent aussi être économiques. Il est en effet essentiel que l'argument technique n'entre pas en contradiction avec l'argument financier.

\section{La question financière}

L'exécution de lignes dites « économiques » sur le réseau français est un problème antérieur au plan Freycinet. La question de l'utilisation de cette filière technique s'est posée avec la promulgation de la loi de $1865^{25}$, mais les ambitions ferroviaires de la Troisième République et, surtout, les précoces difficultés de réalisation que rencontre le troisième réseau ravivent la réflexion chez les ingénieurs parlementaires. L'enjeu financier est d'autant plus crucial que le nouveau réseau, destiné à servir des intérêts locaux, sera à coup sûr d'une faible rentabilité. Il est par conséquent indispensable de l'établir au moindre coût. Aussi, dès 1879, l'ingénieur Léon Vauthier - qui n'est pas parlementaire - milite-t-il en faveur de «l'équilibre de nos budgets » pour « le plus rapide développement possible des voies économiques $»^{26}$. 
La faiblesse du coût d'établissement constitue en effet l'argument essentiel sur lequel s'appuie l'ingénieur des Mines Wickersheimer pour défendre son projet très controversé de chemins de fer sur route. Se fondant sur l'exemple italien, il évalue le coût d'établissement à hauteur de 40 à 50000 francs par kilomètre et son coût d'exploitation serait également modique ${ }^{27}$. Pour la même raison d'économie, Wickersheimer demande le déclassement des lignes secondaires d'intérêt local ou général «qui sont aujourd'hui administrées avec un luxe d'exploitation tout à fait excessif $»^{28}$. Il dépose au cours de la séance suivante un amendement qui vise à diminuer le budget des Travaux publics de dix millions, mais qui est massivement repoussé par 302 voix contre 126, les votes favorables provenant essentiellement du camp radical. Dans ce scrutin, les ingénieurs suivent l'opinion générale de la Chambre en désavouant leur collègue par 11 voix contre 7 . Baihaut, rapporteur du budget des Travaux publics, est le principal adversaire de Wickersheimer en dénonçant cette amputation drastique et injustifiée du budget qui pourrait porter préjudice aux travaux en cours.

Les partisans d'une voie normale en site propre y vont aussi de leur démonstration financière. Krantz, à nouveau, conseille la construction d'une ligne unique d'un rayon minimum de 350 mètres, d'une inclinaison maximale de 15 millimètres par mètre, avec des rails de 35 à 37 kilogrammes. Les économies seraient réalisées par la suppression des barrières aux passages à niveau et des clôtures, la diminution du nombre de stations, le renoncement à la construction d'une deuxième voie parallèle ${ }^{29}$. De cette manière, il serait possible d'obtenir un chemin de fer économique dont il évalue le coût de premier établissement entre 85000 et 110000 francs par kilomètre. Cette évaluation représente certes le double de celle proposée par Wickersheimer pour le chemin de fer sur route. Elle n'en représente pas moins un chemin de fer véritablement économique, quand on sait que Freycinet propose en 1879 un coût de 200000 francs par kilomètre pour l'établissement d'une ligne normale, estimation au demeurant sous-évaluée par rapport à la réalité.

Les rares partisans de la voie étroite chez les ingénieurs parlementaires ne sont pas en reste. Joseph de Gasté, membre du centre gauche, député du Finistère et, de son état, ingénieur du corps de la Marine, intervient une première fois en 1878, au cours de la discussion relative au projet d'incorporation de chemins de fer d'intérêt local dans le réseau d'intérêt général. Il y déplore la principale lacune de la loi de 1865 qui a oublié de fixer la largeur des chemins de fer d'intérêt local. «Si elle avait limité cette largeur à 72 centimètres, regrette-t-il, c'est-à- 
dire à la moitié de la largeur de la voie ordinaire, les frais de terrassement auraient été réduits au quart ; les locomotives auraient pu peser sept tonnes au lieu de trente ; le matériel eut été plus léger, et en conséquence, on aurait fait au prix de 40000 à 60000 francs, matériel roulant compris, le kilomètre de chemin de fer d'intérêt local. Dans ces conditions, presque tous les chemins de fer auraient payé leurs frais $[. . .]^{30} . » \mathrm{Sa}$ deuxième intervention est rédigée. Dans un texte intitulé Quelques réflexions sur le budget de 1883, Gasté vise désormais la conception technique du troisième réseau, alors que le plan Freycinet prend des allures de gouffre financier. "Construits [...] avec la même largeur de voie que les chemins de fer principaux de la France, les nouveaux chemins de fer coûteront quatre fois autant environ que construits à voie étroite : leur recette kilométrique sera inférieure à leur dépense d'exploitation qui s'élèvera, en moyenne, à un minimum de 6000 francs par kilomètre, soit trois fois la dépense d'un chemin de fer à voie étroite. » Dans cette perspective, le pays peut craindre de s'enfoncer dans un maelström financier pour de longues années ${ }^{31}$.

Pour la même raison, à savoir la croissance démesurée du budget extraordinaire, l'ingénieur en chef et député de l'Aisne Jules Lesguillier propose en mai 1881 la construction de 10000 kilomètres à voie réduite établis en pays de montagnes, sur les 17000 que propose le classement de 1878. Ces 10000 kilomètres « coûteront au moins de 300 à 400000 francs par kilomètre et les recettes seront certainement très loin de couvrir les frais d'exploitation $»^{32}$. Aussi, l'ingénieur des Ponts et Chaussées prévoit-il un "sacrifice de quatre milliards à demander au pays ", que seul l'établissement de la voie étroite permettrait de réduire de moitié. Lesguillier ne souhaite pas en fait une réduction du programme Freycinet, mais, au contraire, son extension sur un autre mode. Celle-ci, déclare-til, " est indispensable [...] si nous voulons, comme nous en avons la prétention, marcher à la tête de la civilisation $»^{33}$. Il recommande en particulier la construction de 3000 kilomètres de lignes parallèles aux artères principales mais saturées de l'ancien réseau. L'essentiel pour lui reste néanmoins l'établissement, en dehors du plan Freycinet, de 40000 kilomètres supplémentaires construits à voie étroite. Il dépose une proposition de loi en ce sens le 24 juillet 1882. Parmi les quatre-vingttrois signataires figurent quatre autres ingénieurs : Brossard, Levet, Reymond appartiennent au milieu industriel et des ingénieurs civils; seul Mangon est ingénieur d'État. L'argument décisif est le classement de la France en matière d'équipement ferroviaire. Avec 40000 kilomètres exploités ou classés, elle n'occupe qu'un modeste sixième rang, d'où la nécessité de doubler purement et simplement le réseau. Lesguillier se situe parmi cette catégorie d'ingénieurs qui mesurent la puissance 
économique d'un pays développé au nombre de kilomètres de voies ferrées. Il préconise à cet égard « une voie étroite plus en rapport avec le trafic restreint des lignes dont il s'agit, et qui, en pénétrant mieux au cœur des centres de populations, offre plus d'avantages au point de vue des intérêts locaux ». Cette proposition reste néanmoins sans lendemain.

Au-delà de l'argument strictement budgétaire, on peut enfin déceler chez certains partisans de la voie étroite une mise en accusation, tantôt tacite, tantôt ouverte, des doctrines en vigueur au sein de l'administration des Ponts et Chaussées. Wickersheimer, par exemple, dénonce sa propension à «faire luxueux » et à oublier que «les chemins de fer sont avant tout un instrument commercial $»^{34}$. Mais, en l'occurrence, le terrain de la polémique s'est déjà déplacé.

\section{Conclusion}

Si le débat relatif aux réseaux secondaires tend à s'épuiser à la fin des années 1880 chez les ingénieurs parlementaires, il n'est pas clos pour autant dans le milieu des techniciens en général. On peut ainsi relever l'ouvrage de l'ingénieur en chef des Ponts et Chaussées Félix Martin, publié en 1891, qui constitue un vibrant plaidoyer en faveur du développement de la voie métrique dans la mesure où, précise l'auteur, les chemins de fer qui restent à construire en France sont des lignes agrico$\operatorname{les}^{35}$. Dans ce débat, les lignes de clivage apparaissent avec assez de netteté. L'impératif budgétaire prime toute autre considération chez les défenseurs de la voie étroite, qu'elle soit établie ou non en site propre. A l'inverse, les partisans de la voie normale préfèrent invoquer les avantages techniques, plus importants à leurs yeux, quitte à faire le choix d'une option moins économique. Cette controverse dépasse pourtant la seule mise en balance des arguments financiers et techniques. Elle montre également les conceptions divergentes à l'égard des notions de réseaux d'intérêt local et général. La différence de nature entre les deux réseaux paraît moins évidente chez Krantz ou Varroy, de même que chez Armand Rousseau et Albert Pesson, tous ingénieurs du corps des Ponts et Chaussées favorables à la voie normale, pour lesquels l'homogénéité et la continuité du réseau ferroviaire français sont primordiales. Mais c'est aussi un autre ingénieur de ce corps, Jules Lesguillier, qui recommande une configuration particulière aux réseaux secondaires car il en imagine les fonctions spécifiques. Cette dernière remarque montre également que la controverse relative aux réseaux secondaires chez les ingénieurs parlementaires dépasse parfois, bien qu'elle demeure en grande partie fondée, la simple opposition entre ingénieurs des Ponts et les autres. 


\section{Notes}

1- Cf. Registres de matricules de l'École Polytechnique ; Archives nationales F 14 2251/1 ; Jean-Marie Mayeur ; Alain Corbin, Les Immortels du Sénat, 1875-1918, Paris, Publications de la Sorbonne, 1995, p. 361.

2- Michèle Merger, La Politique de la Troisième République en matière de navigation intérieure (1870-1914), thèse de $3^{\text {e }}$ cycle, université de Dijon, sous la dir. de François Caron, 1979, p. 52 et suiv.

3- Jean-Baptiste Krantz, Observations au sujet des chemins de fer d'intérêt général et local, Paris, Delamotte et fils, 1875, p. 11.

4- Ibid., p. 15.

5- Ibid., p. 14.

6- Ibid., p. 18.

7- Ibid., p. 36.

8- Ibid., p. 36.

9- Ibid., p. 12.

10- Cette formule est permise par l'article 89 de la loi de 1871.

11- J.-B. Krantz, op. cit., p. 85.

12- Alfred Picard, Les Chemins de fer, Paris, Dunod et Pinat, 1918, p. 48.

13- J.-B. Krantz, op. cit., p. 82. Les mots sont soulignés par l'auteur.

14- Ibid., p. 80.

15- J.-B. Krantz, Observations sur les chemins de fer économiques à voie normale et à voie réduite, Paris, Delamotte et fils, 1875, p. 85.

16- Annales de l'Assemblée nationale, t. 38, p. 140. Séance du 20 mai 1875.

17- Impression n 3 343, Annales, annexes, t. 41, p. 181. Séance du 30 juillet 1875.

18- Annales, session extraordinaire 1888, t. unique, p. 677. Séance du 29 novembre 1888 .

19- J.-B. Krantz, op. cit., p. 51.

20- C'était notamment la position défendue par la commission réunie en 1861.

21- J.-B. Krantz, op. cit., p. 28.

22- Ibid., p. 93.

23- Ibid., p. 38.

24- Ibid., p. 4. Krantz justifie néanmoins l'usage de la voie réduite dans certaines régions particulièrement déshéritées comme la Corse, au sol tourmenté et à la faible densité de population, sans véritable industrie ni commerce et surtout dépourvue de routes et de canaux. C'est aussi l'idée d'Armand Rousseau, autre ingénieur des Ponts et député du Finistère, qui déclare en 1882, à propos de la 
voie étroite, « qu'il en peut être fait application pour certaines lignes [...] surtout dans des cas spéciaux, pour les pays de montagnes »; il demeure néanmoins lui aussi persuadé de leur rôle restreint.

25- François Caron, Histoire des chemins de fer en France, t. I, 1740-1883, Paris, Fayard, 1997, p. 436. En 1876, le journal gambettiste La République française fait campagne pour la construction par le secteur privé d'un réseau secondaire à bon marché, remettant ainsi à la mode le « chemin de fer écossais » lancée par Michel Chevalier en 1861. Cf. Marie-Noëlle Thibault, La Question du rachat des chemins de fer dans l'idéologie républicaine au XIX siècle (1843-1883), thèse de $3^{\circledR}$ cycle, université de Dijon, sous la dir. de F. Caron, 1975, p. 175.

26- Léon Vauthier, Projet de réorganisation des chemins de fer français. Projet de loi précédé d'un exposé des motifs, Paris, A. Chaix, 1879, p. 14.

27- Annales, session extraordinaire 1888, t. unique, p. 677.

28-Ibid., p. 676.

29- Sur ce demier point, la doctrine de Krantz évolue entre 1875 et 1879, comme le montre son discours au Sénat. Cf. Annales, t. 8, session ordinaire 1879, p. 87. 30-Annales, t. 4, p. 100. Séance du 15 mars 1878. L'ingénieur et économiste libéral Clément Colson précise qu'un coût de premier établissement aussi faible ne peut être obtenu que dans des conditions très faciles. La voie étroite a néanmoins sa préférence car elle donne à l'entreprise « un caractère plus modeste, ce qui diminue les exigences du public et permet de simplifier le service [...] », bien qu'il constate l'importance non négligeable des frais de transbordement dans les gares de jonction. Cf. Clément Colson, Transports et tarifs, Paris, Rothschild, 1890, p. 267.

31- Joseph de Gasté, Quelques réflexions sur le budget de 1883, p. 3.

32- D'après Sévène, la moyenne d'établissement d'une ligne de 1,44 mètre de large, dans des conditions normales, s'élève à 215000 francs par kilomètre. Cité par C. Colson, op. cit., p. 266.

33- Jules Lesguillier, « La question des chemins de fer et M. Léon Say », L'Écho républicain de l'Aisne, 1882, p. 4-5.

34- A.N. / C 5385. Procès-verbaux de la commission des chemins de fer.

35- Félix Martin, Du régime des chemins de fer secondaires en France, Paris, Librairie polytechnique, Baudry et Cie Éditeurs, 1891, p. 12. 
